\title{
A Fast Packet Switching Satellite Communication Network
}

\author{
Enrico Del Re and Romano Fantacci \\ Dipartimento di Ingegneria Elettronica \\ Universita' di Firenze \\ Via S. Marta 350139 Firenze - Italy
}

\begin{abstract}
A multibeam fast packet switching satellite communication network serving multiple zones is studied in this paper. The synchronous Time Division Multiple Access protocol (TDMA) is use in transmitting messages in the uplink channels while the Asinchronous Time Division Multiple Access protocol (ATDMA) is used in the downlink channels. Moreover, fast packet switching capabilities are assumed on-board the satellite. Alternatives for the architecture of the on-board fast packet switching fabric are considered. The performance of the considered approaches have been derived by theoretical analysis and computer simulations. A novel input queueing technique is also proposed and analyzed to show that it achives better performance with respect to the classical input queueing approach. Therefore, by means of the novel input queueing approach it is possible to lower the end-to-end delay without increasing the complexity of the on-board equipments.
\end{abstract}

\section{INTRODUCTION}

The communication scenario is rapidly changing. The widespread acceptance of the integrated services digital network [1]-[2] (ISDN) is forecast to rise rapidly following the increased user demand for new telecomunication services. Moreover an evolution towards the broadband ISDN with the consequent requirement for efficient multiplexing and switching techniques is presently under study and definition to permit an increased flexibility, a high traffic capacity and an enhanced bandwidth efficiency [3]-[4]. Recently, new solution for the broadband ISDN has been considered in international standard organization like the CCITT. Presently CCITT Recommendation I.121 defines the Asyncronous Transfer Mode (ATM) as the target solution for the future broadband ISDN. Activities are in progress all over the world to implement prototypes based on the ATM technique

Work carried out under the financial support of the National Research Council (C.N.R.) in the frame of the Telecommunication Project.
In this context the role of a communication satellite changes. Instead of being a single repeater, the satellite should operate as a node of a (possibly integrated) space/terrestrial network.

To achieve as far as possible this integration, the space subnetwork architecture has to use access methods and switching techniques compatible with the ATM solution. For example, a suitable architecture is to use a satellite subnetwork with multiple spot beams and with baseband switching capabilities on board, providing the required interconnections among the different beams. An obvious choice is to use the same switching techniques as in the ATM terrestrial networks, i.e. the Fast Packet Switching (FPS) with slotted capacity [4], [14], [16], [20].

We focus here on a network-oriented satellite communication system in which the satellite is a remote node of a complex broadband terrestrial network which includes terrestrial facilities (nodes and trunks). The available capacity of each uplink is shared among the earth stations, which are nodes themselves, located in the same spot beam. Moreover, in the satellite communication network considered in this paper, the satellite coverage area is divided into geographically disjoint zones and a multiple spot beam satellite is used to provide coverage. Therefore, the possidility of the frequency reuse makes the multibeam satellite more efficient than a global beam satellite in terms of spectrum and power utilization.

The main goal of this paper is the evaluation of the end-to-end delay which is formed (apart the round trip delay) by the sum of three contributions. A first contribution is due to the access delay to the satellite by the earth stations. This parameter is strongly dependent on the access protocol used in the up-link which in this paper is assumed to be the TDMA. A second contribution is due to the on board switching delay introduced when two or more packets arrive simultaneously on different inputs with the same output as destination. One of these contending packets attained switching while queueing is required for the other to wait for a later route.

5A.2.1. 
Depending on the speed of the switch fabric and its architecture, different approaches for providing the queueing necessary for contending packets have recently appeared in the scientific literature [4],[14],[16],[20].

This paper is focus on the input queueing. In particular, with the aim to decrease the on-board switching delay, a novel input queueing approach is proposed and studied. Finally, the third contribution is due to the transmission delay into the downlinks. The ATDMA protocol is assumed in transmission onto the downlinks [8],[13]. We are motivated in this choice by the statistical multiplexing of the packets performed in the on-board queue.

The downlink transmission delay is a well known parameter, therefore, its evaluation will be not further considered in this paper. Results for the satellite communication network under consideration will be presented in terms of mean access delay and mean on-board switching delay.

\section{SATELLITE NETWORK MODEL}

The satellite communication system considered in this paper (Fig. 1), has the following characteristics:
1) the entire coverage area is divided into $\mathrm{N}$ geografically disjoint spot beam zones which make it possible frequency reuse in the uplinks;

2) the satellite has on-board baseband processing and switching capabilities;

3) infinite buffers are provided on-board for messages waiting for transmission in the dowlinks;

4) there are $N$ receivers and $N$ transponders on the satellite and at most one transponder can serve the same zone at the same time;

5) each received packet on board the satellite can have as destination any of the possible $\mathbf{N}$ zones with equal probability;

6) the service provided by each transponder has it own frame structure, which is filled by

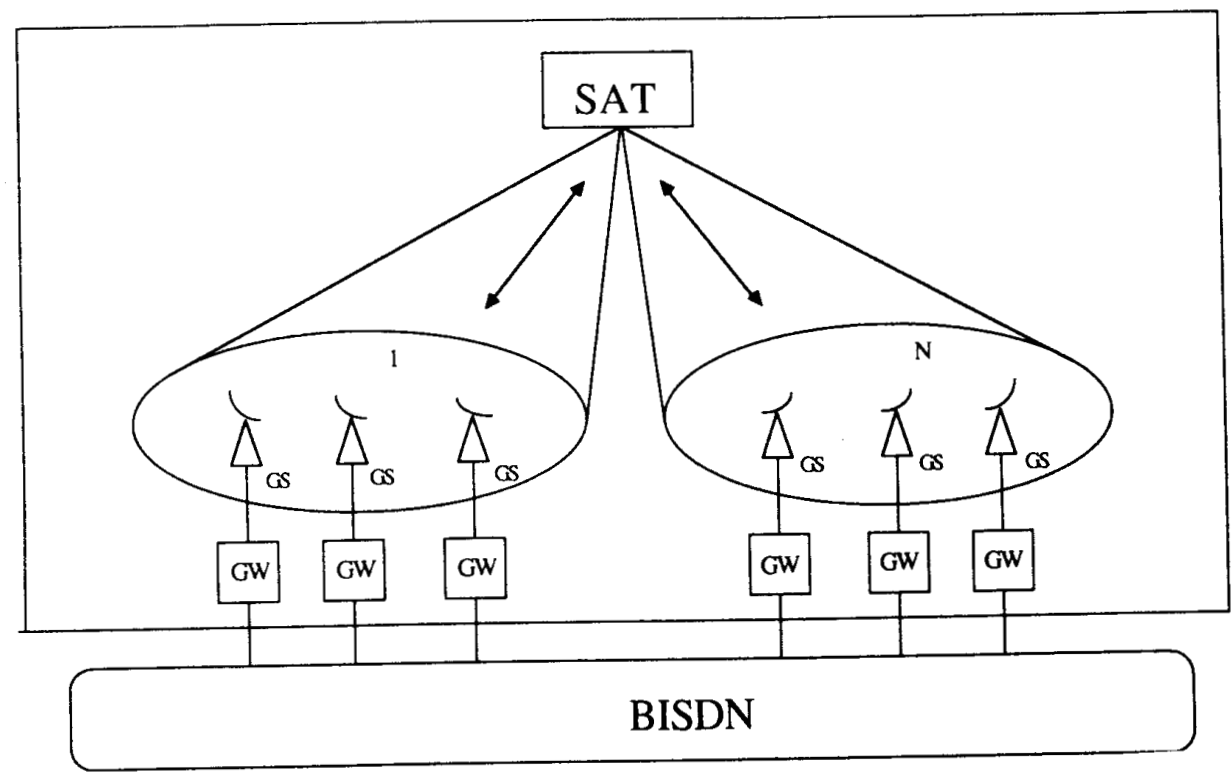

Fig. 1 - General scheme of the multibeam fast packet switching satellite network. $\mathrm{GS}=$ Earth stations $; \mathrm{GW}=$ Gateways $; \mathrm{SAT}=$ Satellite.

5A.2.2. 
transmitting packets (if any) from

the corresponding buffer in a

First-In-First-Out (FIFO) discipline.

At each terrestrial station packets arrive according to a Poisson process with rate $B$ (packets/s). For semplicity it is assumed that each message is formed by a packet of a fixed length.

The overal end-to-end delay for the satellite network under consideration results to be:

$$
T_{t}=T_{U}+T_{B}+2 t_{d}
$$

where:

$T_{U}$ - denotes the total time which elapses from the instant of arrival of the packet at the earth station to the instant of its transmission completion in the uplink channel;

$T_{B}$ - denotes the total time which elapses from the instant of arrival of the packet on-board the satellite up to the instant of its transmission completion in the downlink channel. Note that $T_{B}$ is strongly influenced by the architecture of the on-board FPS fabric;

$t_{d}$ - denotes the round-trip delay.

The parameter $T_{U}$ will be evaluated in the following section while the evaluation of the $T_{B}$ will be the subject of the Sect. III.

\section{UPLINK TDMA PROTOCOL}

In the TDMA protocol time is divided in frame of fixed length. Each frame contains $\mathrm{N}_{\mathrm{s}}$ slot with duration $\tau$.

The theoretical analysis will be done at packet departure times as is commonly used for $\mathrm{M} / \mathrm{G} / 1$ queueing system [8]-[13].

Nevertheless, in our case we must account for the special kind of service. Therefore, the usual analysis must be modified to account for the fact that a packet which arrives at an empty system may not reiceive immediate service [12]. It can be required that such a packet must wait for the next slot. This additional waiting time does not arise for packets arriving at a busy system. The time diagrams for arrivals to a busy and empty system is shown in Fig. 2. The service time of a packet starts when that packet reaches the head of the queue. The service time ends when the packet leaves the sistem. In case of Fig. $2 \mathrm{a}$, the packet labelled as $C_{n}$, arrives at a busy system, and its service time is equal to a frame (i.e. $T_{f}=N_{s} \tau$ ). In case of Fig. $2 b$, the packet $C_{n}$ arrives to an idle system, therefore it reiceves a different service which lasts a fractional frame followed by an integral frame.
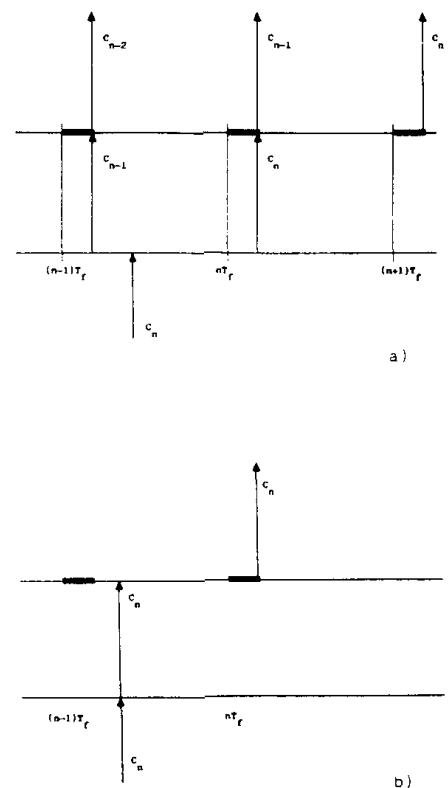

Fig. 2 - Time diagrams for arrivals.

a) busy system ; b) empty system.

It follows that the total delay in transmitting a packet from an arbitrary earth station to its destination can be considered to have three components:

a) the packet transmission time $\tau$;

b) the queueing delay in the buffer of the station;

c) the slot synchronization delay.

The mean value of the slot synchronization delay for Poisson arrivals can be assumed equal to one-half the frame time, i.e. $\mathrm{N}_{\mathbf{s}} \tau / 2$ [12].

To evaluate the mean value of the queue delay, each station can be modeled as an independent $M / D / 1$ queueuing system, [8]-[13], with a mean arrival rate of $\beta$ (packets/s). Hence, the final result for the mean total delay, normalized with respect to $\tau$, is:

$$
T_{U}=1+\frac{N_{s}}{2}+\frac{\beta \tau N_{s}^{2}}{2\left(1-\beta \tau N_{s}\right)}=1+\frac{N_{s}}{2}+\frac{\Gamma N_{s}}{2(1-\Gamma)}
$$

5A.2.3. 
Fig. 3 shows the parameter $T_{U}$ normalized with respect to $\tau$ as a function of $\gamma\left(=\beta \tau \mathrm{N}_{\mathrm{s}}\right.$ for different values of $N_{s}$. In the same figure the simulation results are also reported to validate the theoretical analysis.

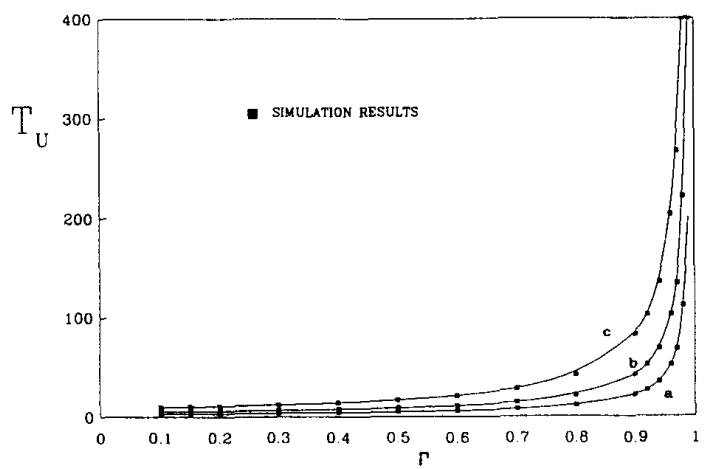

Fig. 3 - Normalized total delay as function of $\mathbf{p}$ for the TDMA protocol.

a) $\mathrm{N}=4$; b) $\mathrm{N}=8$; c) $\mathrm{N}=16$

\section{ON-BOARD FPS FABRIC}

In this section the performance of the on-board FPS fabric are derived by assuming that a fixed number of trasponders, i.e. equal to the satellite spoat beams $\mathrm{N}$, is used.

Independently on the approach used for queueing in the FPS fabric the service time of each packet is constant and equal to $\tau$. Two alternatives are considered in queueing the arrival packets. The classical single queue per input approach is first discussed.

\section{III.1 THE SINGLE QUEUE PER INPUT APPROACH}

The performance analysis presented in this section have been derived by making use of well-known results for discrete-time queueing system [17],[18]. Let us assume that the arrival processes on the $\mathbf{N}$ input links as $\mathrm{N}$ independent Bernoulli process with the probability of an arrival per slot equal to $p$. Each packet has an equal probability to be addressed to any of the other $\mathrm{N}$ output links and successive packets require independent routing. Moreover, we assume that packets are routed to the appropriate output link strictly in their order of arrival which means that the service discipline is FIFO within each input queue. When a packet arrives at the head of its input queue a request asking for routing to the destination output link is sent immediately to the switch controller. For each output link, a queue namely destination queue, is kept by the switch controller. Each new request is placed at the end of the appropriate destination queue. The routing requests are processed on the basis of the FIFO discipline. When a request reaches the head of its queue, the central switch makes the necessary routing between the input and the destination link. No more than one request can be satisfied on each slot. Hence, each destination queue may be considered as a $M / D / 1 / N / N$ queueing system.

By means of the previous considerations it follows immediately that each input queue may be modeled as a discrete $M / G / 1$ queueing system with the service time per packet equal to the total delay spent by the corresponding routing request in the destination queue.

Fixing our attention on a particular input queue, the imbedded Markov chains approach developed for the continuous $\mathrm{M} / \mathrm{G} / 1$ model is applicable here also to derive the mean total delay per packet. The probability generating function of the number of packets in the input queue, assuming equilibrium, is:

$$
Q(z)=\frac{Q_{0} A(z)(z-1)}{z-A(z)}
$$

where $Q_{0}$ is the probability of having an idle queue and $A(z)$ is the probability generating function of the number of arrivals during a service period of a customer. We have also

$$
A(z)=(1-p+p z) G(1-p+p z)
$$

where $G(z)$ is the probability generating function of the waiting time (normalized to $\tau$ ) or equivalently in our case the probability generating function of the total time spent by packets waiting for reaching the

\section{A.2.4.}


head of the destination queue.

In deriving an expression for $\mathrm{G}(\mathrm{z})$ it must be taken into account that in the considered case, the routing requests may arrive to the appropriate destination queue in batches of random size [19], [20]. We assume that routing requests which arrive at the destination queue at a same instant are served in a random order. However, the routing requests arriving in earlier instants, are served first on the basis of the FIFO discipline. Therefore, the total time spent in the destination queue waiting for service by a routing request is due to the sum of two contributions, e.g. $w_{1}$ and $w_{2}$. The term $w_{1}$ takes into account the time necessary to serve all the routing requests which are waiting in the queue at the arrival instant. The second term $\mathrm{w}_{2}$, is an additional delay due to the service of the routing requests which arrived at the same instant and were randomly selected to be served first.

Focusing on a destination queue (the tagged destination queue), and assuming that $\mathrm{k}$ packets are already waiting for routing, the probability that a routing request (the tagged routing request) arrives in a batch of size $i$ is given by :

$$
P(i / k)=\frac{i}{(N-k) \alpha}\left(\begin{array}{c}
N-k \\
i
\end{array}\right) \alpha^{i}(1-\alpha)^{N-k-i}
$$

where $\alpha$ is the probability of having a packet from one of the input queues requesting routing to the tagged output link.

In deriving an expression for $\alpha$ we define:

- $A_{m}$ as the overall number of routing requests arrived at all the destination queues at the mth time slot;

- $F_{m}$ as the number of free input queues at the $m$ th time slot.

According to our assumptions, an input queue is free at the mth time slot if it is idle or if the packet at its head has been selected to be routed during the $(\mathrm{m}-1)$ th time slot. It is evident that an arrival at a destination queue must come only from a free input queue. It follows that:

$$
P\left\{A_{m}=j\right\}=\left(\begin{array}{c}
F_{m} \\
j
\end{array}\right)(N \alpha)^{j}(1-N \alpha)^{F_{m}}
$$

Therefore, the mean number of arrivals results to be:

$$
A_{m}\left(F_{m}\right)=F_{m} N \alpha
$$

Let $B_{m}$ be the number of routing requests in all the destination queues, that are waiting for service, $F_{m}$ results to be:

$$
F_{m}=N-B_{m}
$$

In a steady state condition we have:

$$
\bar{F}=N-\bar{B}
$$

where $\overline{\mathrm{F}}$ denotes the mean number of free input queues and $\bar{B}$ is the mean number of routing requests in all the destination queues.

By assuming equilibrium we also have:

$$
A=[N-\bar{B}] N \alpha=N p
$$

For the last expression of (10) it must be noted that the mean number of routing requests which arrive at all the destination queues per time slot, equal to the mean number of packets routed from input queues to the output links, is equal to the mean number of packets which arrive at all the input queues.

It follows from $(9),(10)$ that:

$$
\alpha=\frac{p}{N-\bar{B}}
$$

By assuming $\bar{B}$ small with respect to $N$ we have:

$$
\alpha=p / N
$$

5A.2.5. 
The probability generating function of the waiting time, conditioned on the occurrence that $k$ request are waiting for routing in the tagged destination queue and on the event that the tagged request arrives in a batch of size $i$ is:

$$
G(z / i, k)-\sum_{j=0}^{i-1} \frac{z^{j+k}}{i}=\frac{1-z^{i}}{i(1-z)} z^{k}
$$

Therefore, $G(z / k)$ is given by:

$$
\begin{gathered}
G(z / k)-\sum_{i=1}^{N-k} G(z / i, k) P(i / k)= \\
\frac{1-(1-\alpha+\alpha z)^{N-k} z^{k}}{(N-k) \alpha(1-z)}
\end{gathered}
$$

The probability $P_{R}(k)$ of having $k$ routing requests $(0 \leq \mathrm{k} \leq \mathrm{N}-1)$ in the destination queue can be obtained numerically by an application of the Markov chain balance equations. Fig. 4 shows an example of the Markov chain to be considered when $N=4$. The final results is:

$$
\begin{gathered}
P_{R}(1)-P_{R}(0) \frac{\left(1-a_{0,0}-a_{0,1}\right)}{a_{1,0}} \\
P_{R}(k)=\frac{1-a_{k-1,1}}{a_{k, 0}} P_{R}(k-1)-\sum_{i=2}^{k} \frac{a_{k-i, i}}{a_{k, 0}} P_{R}(k-i)
\end{gathered}
$$

where $2 \leq k \leq N-1$

with $\mathrm{P}_{\mathrm{R}}(0)$ determined in order to verify the following equation:

$$
\sum_{k=0}^{N-1} P_{R}(k)=1
$$

and the terms $a_{i, j}$ (Fig. 4), given by:

$$
a_{i, j}=\left(\begin{array}{c}
N-i \\
j
\end{array}\right) \alpha^{j}(1-\alpha)^{N-i-j}
$$

Therefore, the probability generating function of the waiting time $\mathrm{G}(\mathrm{z})$ can be derived as :

$$
\begin{aligned}
& G(z)-\sum_{k-0}^{N-1} G(z / k) P_{R}(k)= \\
& \sum_{k=0}^{N-1} \frac{\left[1-(1-\alpha+\alpha z)^{N-1-k}\right] z^{k}}{(N-1-k) \alpha(1-z)} P_{R}(k)
\end{aligned}
$$

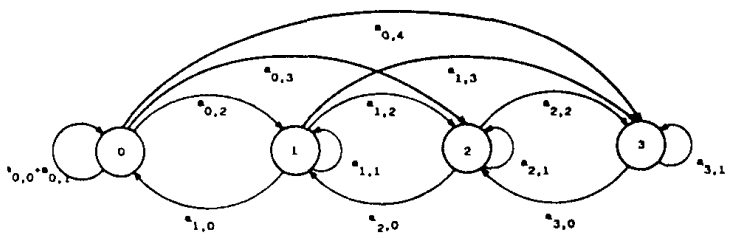

Fig. 4 -The discrete Markov chain $(\mathrm{N}=4)$.

The mean delay spent by a packet in an input queue results in:

$$
T_{B}=\frac{Q^{\prime}(1)}{p}=\frac{A^{\prime}(1)}{p}+\frac{A^{\prime \prime}(1)}{2 p\left[1-A^{\prime}(1)\right]}
$$

where $A^{\prime}(1)$ and $A^{\prime \prime}(1)$ are obtained by differentianting one and two times $A(z)$ with respect to $\mathrm{z}$ and taking the limit as $\mathrm{z}$ approaches 1 . The final result is:

$$
T_{B}=1+\sum_{k=0}^{N-1} \frac{(N+k-1) \alpha}{2} P_{R}(k)+
$$

$$
p \frac{\sum_{k=0}^{N-1} k[k-1+\alpha(N-k-1)] P_{R}(k)}{\left.2-p\left[2+\sum_{k=0}^{N-1}(N-k-1) \alpha P_{R}(k)\right)\right]}+
$$

$$
\left.\frac{\sum_{k=0}^{N-1}(N-k-1)(N-k-2) \alpha^{2} P_{R}(k)}{3\left\lfloor 2-p\left[2+\sum_{k=0}^{N-1}(N+k-1) \alpha P_{R}(k)\right]\right\}}\right\}(21)
$$

5A.2.6. 
Fig. 5 shows $\mathrm{T}_{\mathrm{B}}$, (normalized with respect to $\tau$ ), as a function of $p$ for different values of $N$. In the same figure the results derived by computer simulations are reported to validate the analytical results. From this figure, it can be pointed out that the analytical results agree with the simulation results for small and medium value of $\mathrm{p}$. However, for values of $\mathrm{p}$ approaching the maximum achievable throughput our analysis leads to a slight underestimation. This is the effect of using the approximation of the parameter $\alpha$ in (12) instead of the exact value (11).

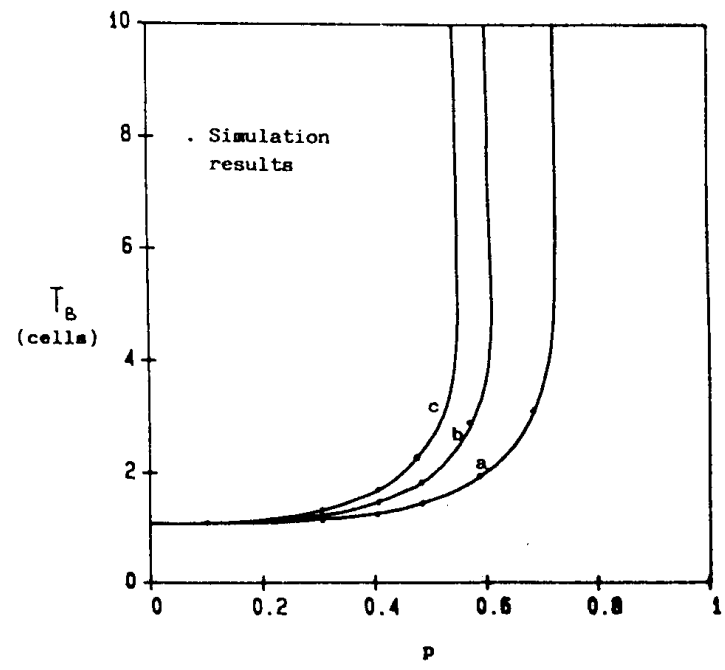

Fig. 5 - The mean normalized total delay for input queueing with FIFO selection policy.

a) $\mathrm{N}=2$; b) $\mathrm{N}=4$; c) $\mathrm{N}=16$.

\section{2 THE MULTIPLE QUEUES PER INPUT APPROACH}

In the case of a single queue per input none of the packets waiting for routing in an input queue can leave the queue until the packet at the head has complete service. Conversely, the novel proposed multiple input queueing approach always permits service for packets in input queues whose destination queues are empty.

In the multiple queueing approach, each input queue is splitted in $\mathbf{N}$ separate queues, one for each possible output link. Any new arrived packet is stored in one of these $\mathrm{N}$ queues according to its routing request.
Packets at the head of input queues for the same output link are contenting for routing. The FIFO selection policy is used to select the packet which attains routing, moreover the service discipline in each input queue is FIFO.

In deriving $\alpha$ we focus on a particular output link. The rate of the Bernoulli arrival process at each input queue is now $p / N$. Letting $Q_{m}$ denote the number of routing requests in the tagged destination queue, we can write:

$$
F_{m}=N-Q_{m}
$$

Therefore, in a steady state condition:

$$
\bar{F}=N-\bar{Q}
$$

where the mean number of routing requests in the tagged destination queue can be derived as:

$$
\bar{Q}=\sum_{n=0}^{N-1} n P_{R}(n)
$$

It is straightforward to derive in this case an expression symilar to (10) that now reads as:

$$
(N-\bar{Q}) N \alpha=N p
$$

Hence:

$$
\alpha=\frac{p}{N-\sum_{n=0}^{N-1} n P_{R}(n)}
$$

Eq. (26) defines a non-linear equation in $\alpha$. Solving this equation numerically it is possible to determine $\alpha$.

Starting from the previous considerations, it is straighforward to derive the mean delay per packet for the FIFO selection policy as:

$$
T_{B}=\frac{N A^{\prime}(1)}{p}+\frac{N A^{\prime \prime}(1)}{2 p\left[1-A^{\prime}(1)\right]}=
$$

\section{A.2.7.}




$$
\begin{gathered}
p\left\{\frac{1+\sum_{k=0}^{N-1} \frac{(N+k-1) \alpha}{2} P_{R}(k)+}{\left.2 N-p\left[2+\sum_{k=0}^{N-1}(N-k-1) \alpha P_{R}(k)\right)\right]}+\right. \\
\left.\frac{\sum_{k=0}^{N-1}(N-k-1)(N-k-2) \alpha^{2} P_{R}(k)}{3\left\{2 N-p\left[2+\sum_{k=0}^{N-1}(N+k-1) \alpha P_{R}(k)\right]\right\}}\right\}
\end{gathered}
$$

Fig. 6 shows $T_{B}$ as a function of $p$ for different values of $\mathrm{N}$. It is evident in this figure that the maximum possible throughput now approaches 1 as $p$ approaches 1 . Therefore, it has been shown the superior performance of the proposed queueing approach with respect to that previously considered (Fig. 5).

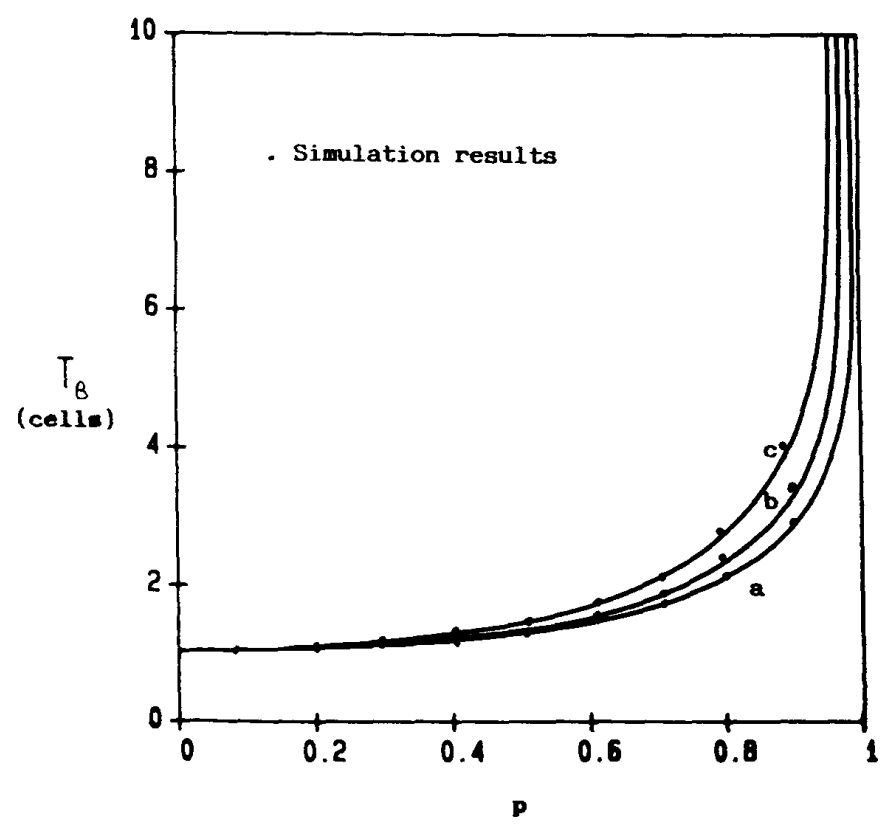

Fig. 6 - The mean normalized total delay for the proposed input queueing approach with FIFO selection policy for different values of $\mathrm{N}$.

$$
\text { a) } \mathrm{N}=2 \text {; b) } \mathrm{N}=4 \text {; c) } \mathrm{N}=16 \text {. }
$$

Fig. 7 shows $T_{B}$ as a function of $p$ for the queueing on inputs and for the proposed multiple queueing on inputs in comparison with that obtained by using the output queueing approach [14], [20]. It is evident in this figure that the proposed multiple queueing on inputs achieves the same performance as the output queueing approach without resorting to a more complex switching fabric [14], [20].

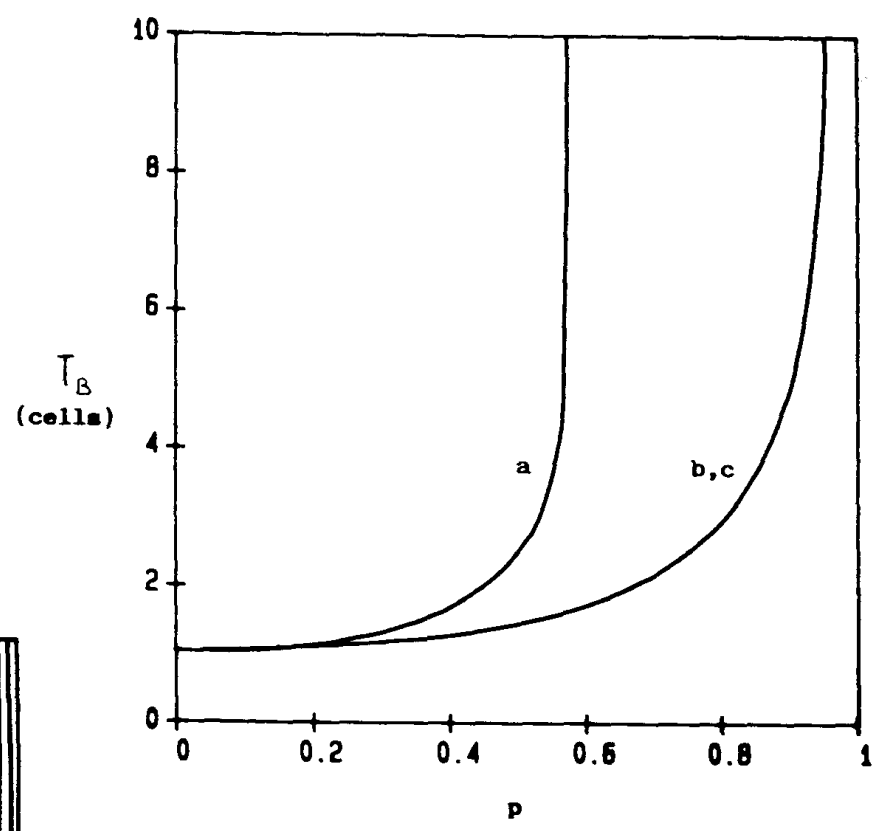

Fig. 7 - Mean normalized total delay as function of $p$ for $\mathrm{N}=16$.

a) queueing on inputs with FIFO selection policy;

b) queueing on outputs;

c) proposed multiple queueing on inputs with FIFO selection policy.

\section{CONCLUSIONS}

In this paper a FPS satellite network has been described and analyzed. The performance of different architectures of the on-board FPS system have been derived. In particular, a novel input queueing approach has been also proposed and analyzed.

The attained results clearly show that the novel input queueing approach outperforms the classical input queueing approach. An important result is that the same performance as the output queueing can be achieved through the use of the proposed technique, without requiring a switch fabric which runs $\mathrm{N}$ times

5A.2.8. 
faster as the input and output links [14], [20]. This results is of particular interest for the fast packet switching satellite communication networks under consideration because it makes it possible to reduce the on board switching delay without increasing the complexity of the on-board equipments.

This paper gives a quantitative analysis for all the contribution to the total end-to-end packet delay. In particular it comes that the delay on board the satellite $\left(T_{B}\right)$ is negligible with respect to the access delay at the ground station $\left(\mathrm{T}_{\mathrm{U}}\right)$. This results indicated a good performance of the satellite switching operations and suggest to improve the access technique to the satellite from the ground stations.

\section{REFERENCES}

[1]J. S. Turner, "Design of an Integrated Services Packet Network", IEEE J. Select. Areas Commun., vol. SAC - 4, pp 1373-1380, Nov. 1986.

[2]J. S. Turner, L. F. Wyatt, "A Packet Network Architecture for Integrated Services", in Proc. IEEE Globecom '83, pp. 45- 50, Dec. 1983.

[3]P. Newman, "A Fast Packet Switch for Integrated Service Backbone Network", J. Select Areas Commun., Vol. Sac - 6, pp. 1468-1479, Dec. 1988.

[4]H. Ahmadi, W. E. Denzel, "A Survey of Nodern High - Performance Switching Techniques", J. Select. Areas Commun., vol. 7, pp. 1091 - 1103, Sept. 1989.

[5]G. Benelli, E. Del Re, R. Fantacci, F. Mandelli, "Performance of Uplink Random - Access and Downlink TDMA Techniques for packet Satellite Networks", IEEE Proceedings, vol. 72, pp 1583, Nov, 1984.

[6]S. Tasaka, "Multiple - Access Protocols for Satellite Packet Communication Networks: A Performance Comparison", IEEE Proceedings, vol. 72, pp. 1573 - 1582, Nov. 1984.

[7]J. F. Chang, "A Multibeam Pachet Satellite Using Random Access Techniques", IEEE Trans. Commun., vol. COM - 31, pp. 1143 - 1154, Oct. 1983.
[8]L. Kleinrock, "Queueing System", vol. 1, New York, Wiley, 1975.

[9]J. F. Hayes, "Modeling and Analysis of Computer Communications Networks", New York, Plenum Press, 1984.

[10]M. Schwartz, "Telecomunication Network: Protocols, Modeling and Analysis", Reading, Massachusetts, U.S.A., Addison - Wesley Publishing Company, 1987.

[11]D. Bertsekas, R. Kallagar, "Data Network", Engleewood Cliffs, New Jersey, Prentice Hall, 1987.

[12]J. L. Hammond, P. J. P. O'Reilly, "Performance Analysis of Local Computer Networks", Reading, Massachusetts, U.S.A., Addison - Wesley Publishing Company, 1986.

[13]A. S. Tanenbaum, "Computer Networks", Englewood Cliffs, NJ. Prentice - Hall, 1989.

[14]M.J. Karol, M.G. Hluchyj, S.P. Morgan, " Input Versus Output Queueing on a Space-Division Packet Switch", IEEE Trans. on Commun., Vol. COM-35, NO. 12, pp. 1347-1356, Dec. 1987.

[15]J.Y. Hui, E. Arthurs, " A Broadband Packet Switch for Integrated Transport", IEEE J. Select. Areas Commun., Vol.SAC-5, NO. 8, pp.264-1273, Oct. 1987.

[16]M.G. Hluchyj, M.J. Karol, " Queueing in High-Performance Packet Switching", IEEE J. Select. Areas Commun., Vol. SAC-6, NO. 9, pp. 1587-1597, Dec. 1988.

[17]T. Meisling, " Discrete-Time Queueing Theory", Oper. Res., Vol. 6, pp.99-105, Jan-Feb. 1958.

[18]H. Kobayashi, A.G. Konheim, " Queueing Models for Computer Communications System Analysis", IEEE Trans. on Commun., Vol. COM-25, NO. 1, pp. 2-28, Jan. 1977.

[19]P.J. Burke, "Delays in Single-Server Queues with Batch Input", Oper. Res., Vol. 23, pp. 830-833, July-Aug. 1975.

[20]E.Del Re, R.Fantacci, "Performance Evaluation of Input Queueing Techniques in ATM Switching Systems". submitted to IEEE Trans. on Comm.

5A.2.9. 